\title{
Identifying unstable modes in stratified shear layers
}

\author{
J. R. Carpenter, ${ }^{1, a)}$ N. J. Balmforth, ${ }^{2}$ and G. A. Lawrence ${ }^{1}$ \\ ${ }^{1}$ Department of Civil Engineering, University of British Columbia, British Columbia V6T 1Z4, Canada \\ ${ }^{2}$ Department of Mathematics and Department of Earth and Ocean Sciences, University of British Columbia, \\ British Columbia V6T 1Z4, Canada
}

(Received 8 July 2009; accepted 26 February 2010; published online 11 May 2010)

\begin{abstract}
One interpretation of the mechanism of instability in stratified shear flows is based on the idea that two independently propagating waves may interact to cause mutual growth in one another. This theory is used in the present study to develop a diagnostic that can be used to identify different types of unstable modes. We focus on stratified shear layers that are susceptible to both the KelvinHelmholtz (KH) and Holmboe $(\mathrm{H})$ modes of instability - though the formulation is more general. The diagnostic is found to be useful in differentiating between $\mathrm{KH}$ and $\mathrm{H}$ modes in the symmetric stratified shear layer (where the center of the shear layer and the density interface coincide). The asymmetric stratified shear layer is also examined since there is no clear distinction between KHand H-type modes in this flow. The KH mechanism of growth is predicted to extend to stronger stratifications (i.e., larger bulk Richardson numbers) than in the symmetric case, in qualitative agreement with nonlinear numerical results. However, the transition is found to be a gradual one in which the $\mathrm{KH}$ mechanism gives way to the $\mathrm{H}$ as the bulk Richardson number is increased. In order to demonstrate the utility of the method, we apply it to instability observed in the Fraser River estuary. () 2010 American Institute of Physics. [doi:10.1063/1.3379845]
\end{abstract}

\section{INTRODUCTION}

When considering the possibility of turbulence production and mixing in a sheared density stratified environment, it is important to determine whether or not a particular flow configuration represents a stable solution of the equations of motion. This problem has a long history that started with the work of Helmholtz ${ }^{1}$ and $\mathrm{Kelvin}^{2}$ on the stability of homogeneous and stratified vortex sheets in the 19th century. Since then, numerous authors have examined cases of increasing complexity in an attempt to understand the basic instability mechanisms that are present in flows with greater physical relevance.

Significant progress was made by Rayleigh ${ }^{3}$ who examined a piecewise-linear representation of the homogeneous shear layer, denoted by $U(z)$, which consists of a finite shear thickness $h$ (see Fig. 1). A stability analysis on this idealized shear layer produced results that are in qualitative agreement with subsequent studies of smooth profiles, such as the hyperbolic tangent shear layer sketched in Fig. $1{ }^{4}$ This suggests that the piecewise-linear shear layer is sufficient to capture the basic instability mechanism that is also present in more realistic smooth profiles. Motivated by geophysical flows, Holmboe ${ }^{5}$ extended Rayleigh's analysis to include a stable density stratification. The piecewise-linear shear layer was retained and a layered, piecewise-constant density profile $\bar{\rho}(z)$ was added with a step change in density of $\Delta \rho$ at the shear layer center (Fig. 1, with $d=0$ ). As in the homogeneous (Rayleigh) case, these idealized profiles give qualitatively similar results to the smooth profiles shown in Fig. $1 .{ }^{6}$ In

\footnotetext{
${ }^{a)}$ Electronic mail: jeffcarp@gmail.com. Present address: EAWAG, Swiss Federal Institute of Aquatic Science and Technology, Seestrasse 79, Kastanienbaum, Switzerland.
}

representing Holmboe's 5 piecewise profiles with smooth functions, it is necessary to introduce another length scale, given by the density interface thickness $\delta$. Qualitative agreement between the smooth and piecewise profiles is achieved when $\delta$ is sufficiently small (i.e., $R \equiv h / \delta \geq 3$, in general) with the piecewise $\bar{\rho}$-profile representing the limit of vanishing $\delta$.

Holmboe's stability analysis shows the presence of two distinct types of unstable modes. Which mode of instability develops is found to be dependent on the wave number $k$, made dimensionless by $\alpha \equiv k h / 2$, and the relative strength of the stratification, measured by the dimensionless bulk Richardson number $J \equiv g^{\prime} h /(\Delta U)^{2}$, where $g^{\prime}=\Delta \rho g / \rho_{0}$ is the reduced gravity, and $\rho_{0}$ a reference density. The resulting stability diagram is shown in Fig. 2(a) for smooth "tanh" profiles with $R=5$. At low $J$, the stratification is relatively unimportant, and the resulting mode of instability is essentially a stratified analogue of the Rayleigh instability. It is common in the literature to refer to this mode as KelvinHelmholtz (KH), despite the closer association with Rayleigh's shear layer, and we keep with this convention throughout. When $J$ is sufficiently large, the qualitative behavior of the instability changes. Unstable Holmboe $(\mathrm{H})$ modes develop due to a destabilizing influence of the stratification, ${ }^{5,7}$ reaching a peak growth rate at finite $J$.

This distinct change in the stability properties that occurs across the KH-H transition can also be seen in the nonlinear development of the instabilities. ${ }^{8-10}$ Figures 2(b)-2(d) show plots of the density and vorticity fields for $\mathrm{KH}$ and $\mathrm{H}$ instabilities, taken from a series of direct numerical simulations (DNS), once they have reached a large amplitude nonlinear stage of development. For details of the simulations see Appendix A. The KH instability [Fig. 2(b)] exhibits the well- 

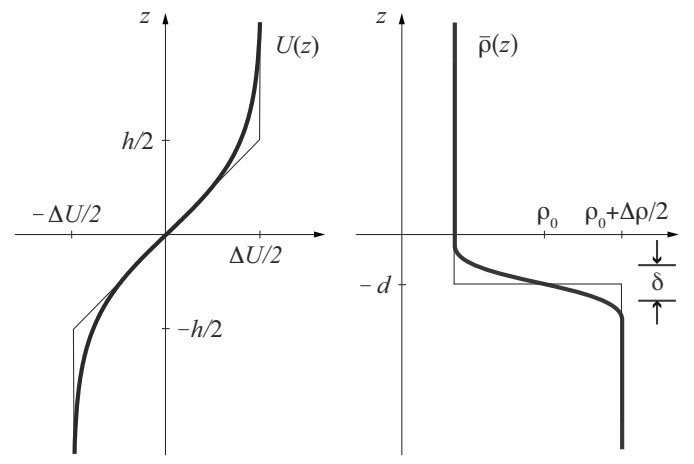

FIG. 1. Profiles of the stratified shear layers to be considered. The thick lines indicate the smooth profiles and the thin lines represent the piecewise profiles.

known billows of overturning fluid caused by a rolling up of the shear layer vorticity (as in the homogeneous shear layer). These billows become susceptible to secondary instabilities and subsequently breakdown to drive turbulent mixing of the density field. ${ }^{9,11,12}$ The nonlinear form of $\mathrm{H}$ instabilities [Figs. 2(c) and 2(d)] consists of cusplike propagating waves that protrude into the upper and lower layers. These generally do not involve a complete overturning of the density interface. It is perhaps not surprising that this different finite amplitude behavior between the $\mathrm{KH}$ and $\mathrm{H}$ modes has recently been found to have a pronounced effect on the mixing of mass and momentum in the shear layer; in some cases changing the effective diffusivity by an order of magnitude across the KH-H transition. ${ }^{13}$ Not only the amount of mixing, but also the character of the mixing has been found to depend on the resulting mode type. The KH instability focuses mixing within the density interface, often tending to produce an intermediate density layer, whereas the $\mathrm{H}$ instability focuses mixing and turbulence on either side of the interface. ${ }^{12,14}$ It is therefore important to predict which mode of instability is to occur when quantifying mixing and momentum transfers.

An important feature of Holmboe's profiles is that they exhibit a symmetry about the shear layer center (once the Boussinesq approximation has been made). However, observations of stratified shear instabilities in the field often display some type of asymmetry. This asymmetry can take many forms such as a vertically displaced density interface relative to the shear layer center, a different proximity of the upper and lower boundaries, and different velocity profile curvatures above and below the density interface, to name a few. For reasons of brevity we shall specifically focus on asymmetry between the shear layer center and the vertical location of the density interface, and refer to it simply as "asymmetry." This asymmetry has been observed in the field, ${ }^{15-18}$ and is also common in laboratory experiments. ${ }^{19-21}$

The implications that asymmetry has on the stability of the flow was first studied by Lawrence et al. ${ }^{20}$ and subsequently by Caulfield et al. ${ }^{22}$ Using the piecewise model of Holmboe $^{5}$ with a density interface located a distance $d$ below the shear layer center, as shown in Fig. 1, Lawrence et al. ${ }^{20}$ found that two distinct branches of instability were present, each consisting of propagating modes. No distinct transition
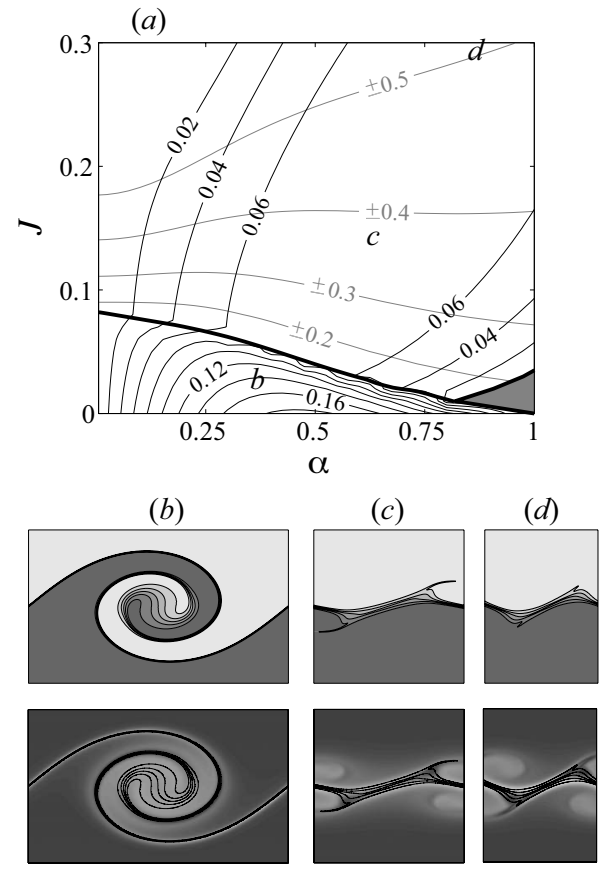

FIG. 2. Stability diagram (a) of the Holmboe model of the stratified shear layer for smooth ( $\tanh$ ) profiles with $R=5$. Dark contours are of growth rate and gray contours of phase speed with the dark gray shading representing the region of stability. The thick lines correspond to the stability boundaries and the transition between stationary (below) and propagating (above) modes. Representative density fields (upper plot) and vorticity fields (lower plot) from DNS are shown in [(b)-(d)]. The approximate location of the instabilities on the stability diagram is indicated with letters. The different widths of $[(b)-(d)]$ are due to changes in the wave number of maximum growth rate. The vertical domain height is taken to be $10 \mathrm{~h}$ for the stability diagram and in the DNS, which is sufficiently large to approximate unbounded domains.

from $\mathrm{KH}$ to $\mathrm{H}$ modes is apparent. One of the two modes always consists of larger growth rates than the other (referred to as the dominant mode), and has often been found to be the only mode observed at large amplitudes. ${ }^{20,23}$ Stability results for the dominant mode of the asymmetric stratified shear layer using smooth profiles with $R=5$, and the asymmetry parameter $a \equiv 2 d / h=0.5$, are shown in Fig. 3. In general, no distinct transition between $\mathrm{KH}$ and $\mathrm{H}$ modes is obvious from the results of linear stability theory when an asymmetry is present $(a \neq 0)$. The asymmetric laboratory observations of Lawrence et al. ${ }^{20,24}$ show a continuous change in behavior from overturning billows to cusplike waves. This can also be seen in the simulation results shown in Figs. 3(b)-3(d), where instabilities resemble $\mathrm{KH}$ at low $J$, and become more like $\mathrm{H}$ instabilities at larger $J$.

The lack of any distinct transition in the stability properties when the flow is asymmetric demonstrates the difficulty in distinguishing between $\mathrm{KH}$ - and H-like instabilities in this case, and raises the question of how to appropriately define what is meant by $\mathrm{KH}$ and $\mathrm{H}$ modes. When the flow is perfectly symmetric the marginal curve separating stationary modes from propagating modes also coincides well with distinct changes in growth rate. These changes in stability characteristics have been found to match reasonably well with changes in the nonlinear behavior of the instabilities, with stationary billows occurring in the $\mathrm{KH}$ region and cusplike 


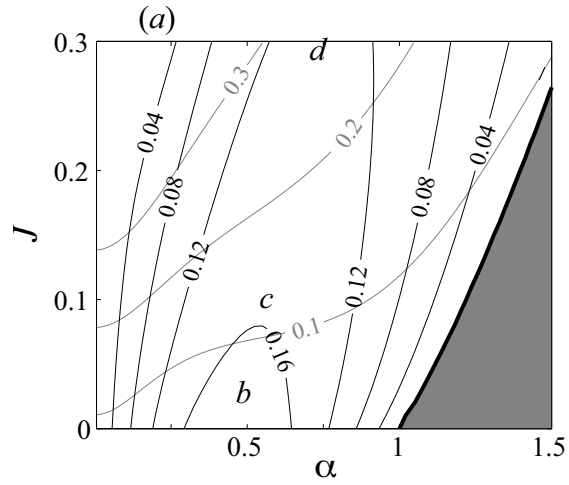

(b)
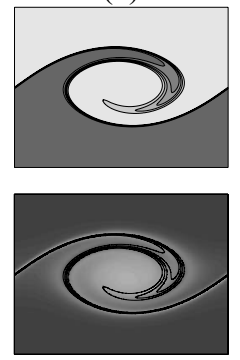

(c)
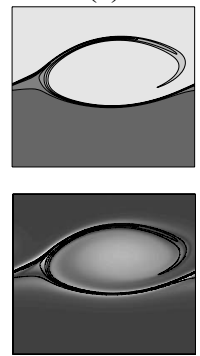

$(d)$

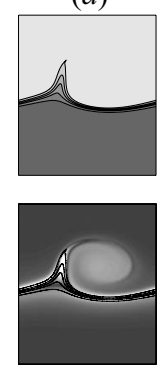

FIG. 3. Stability diagram of the asymmetric stratified shear layer for smooth profiles with $R=5$ and $a=0.5$. See caption of Fig. 2 for further details.

propagating waves in the $\mathrm{H}$ region. It should be noted, however that nonlinear behavior characteristic of both types of instabilities has been observed near the transition between the two mode types. ${ }^{9,10}$ The fact that a transition between $\mathrm{KH}$ and $\mathrm{H}$ behavior, based on the nonlinear dynamics, is observed in asymmetric instabilities, suggests that we must reexamine the distinction based on phase speed between the two modes. We therefore develop a diagnostic to interpret the unstable modes of asymmetric stratified shear layers.

The purpose of this paper is to utilize linear theory to predict the occurrence of $\mathrm{KH}$ - and H-type modes in asymmetric flows where the distinction between them is blurred. The motivation for this is based on three previous findings:

(1) $\mathrm{KH}$ and $\mathrm{H}$ instabilities result from two different linear growth mechanisms (to be discussed fully in Sec. II) $;, 25,26$

(2) it is common to find asymmetry in geophysically relevant flows; ${ }^{15-18}$ and

(3) the type of instability that develops can have a significant influence on turbulent mixing and vertical transports. ${ }^{13,14,27}$

We use results from a linear stability analysis of both piecewise and smooth profiles of the asymmetric stratified shear layer to predict the occurrence of $\mathrm{KH}$ and $\mathrm{H}$ modes. These results are based on the "wave interaction" interpretation of shear instability that is reviewed in Sec. II. This is followed by the formulation of a diagnostic in Sec. III that is used to distinguish between the contributions that $\mathrm{KH}$ and $\mathrm{H}$ modes make to the instability of the flow. Results of applying the diagnostic to the symmetric $(a=0)$ and asymmetric $(a \neq 0)$ cases shown above are outlined and discussed in Sec. IV. We then examine a set of profiles measured from a highly stratified estuary in order to illustrate the applicability of the formulation in a geophysical context. Conclusions are stated in Sec. VI.

\section{WAVE INTERACTION INTERPRETATION OF INSTABILITY}

The wave interaction interpretation attributes instability in stratified and homogeneous shear flows to a mutual interaction between otherwise freely propagating stable waves in the profiles. The majority of work that has utilized the wave interaction interpretation has been performed on the idealized piecewise-linear representation of the stratified shear layer, $5,26,28,29$ since this is the easiest possible geometry to understand and apply the theory. Piecewise profiles of $U$ and $\bar{\rho}$ are particularly simple because they have delta function behavior of vorticity gradients $U^{\prime \prime}$, and density gradients, represented by $N^{2}=-g \bar{\rho}^{\prime} / \rho_{0}$, where primes denote differentiation with respect to $z$. At these locations, referred to as interfaces, wave motion may occur. When isolated from one another, the phase speed of waves on vorticity and density interfaces is given by

$$
c_{v}=U\left(z_{v}\right)+\frac{\Delta q}{2 k} \quad \text { and } \quad c_{d}=U\left(z_{d}\right) \pm\left(\frac{g^{\prime}}{2 k}\right)^{1 / 2},
$$

respectively, where $\Delta q=U^{\prime}\left(z_{v}^{+}\right)-U^{\prime}\left(z_{v}^{-}\right)$denotes the jump in vorticity across the vorticity interface. Note that the vorticity interface supports a single unidirectional mode of propagation, whereas the density interface supports two oppositely propagating modes.

The wave interaction interpretation requires that two interfaces must be present, each supporting an oppositely propagating wave mode, in order for instability to be possible. The two interfacial waves are then able to interact such that (i) they are stationary relative to one another, and (ii) in a "phase-locked" position such that they may cause mutual growth. It is only possible for (i) to occur between two oppositely propagating wave modes when there is shear in the background profile. Condition (i) suggests that the region of instability in the $\alpha J$-plane should be close to the locus of points where the two freely propagating interfacial wave modes have equal phase speeds. Although this is not strictly true, as each wave will interact and adjust the others phase speed, in the limit of large $\alpha$ this interaction vanishes and the approximation becomes accurate. This "resonance" approximation has proven useful in identifying different instability modes in previous studies, ${ }^{25,26,29}$ and reduces to the asymptotic large- $\alpha$ approximation used by Caulfield ${ }^{26}$ and Caulfield et al. $^{22}$

The resonance approximation may be used to identify the wave interactions that lead to instability, and provides an interpretation of the $\mathrm{KH}$ and $\mathrm{H}$ modes that are observed in the piecewise symmetric stratified shear layer profiles of Holmboe. ${ }^{5}$ Figure 4(a) shows the resulting stability diagram with the resonance approximation as a dashed line. The curve is obtained by equating the upper (lower) vorticity wave speed with the rightward (leftward) propagating internal wave speed on the density interface. This results in two curves that are coincident (due to the symmetry) that 
(a)

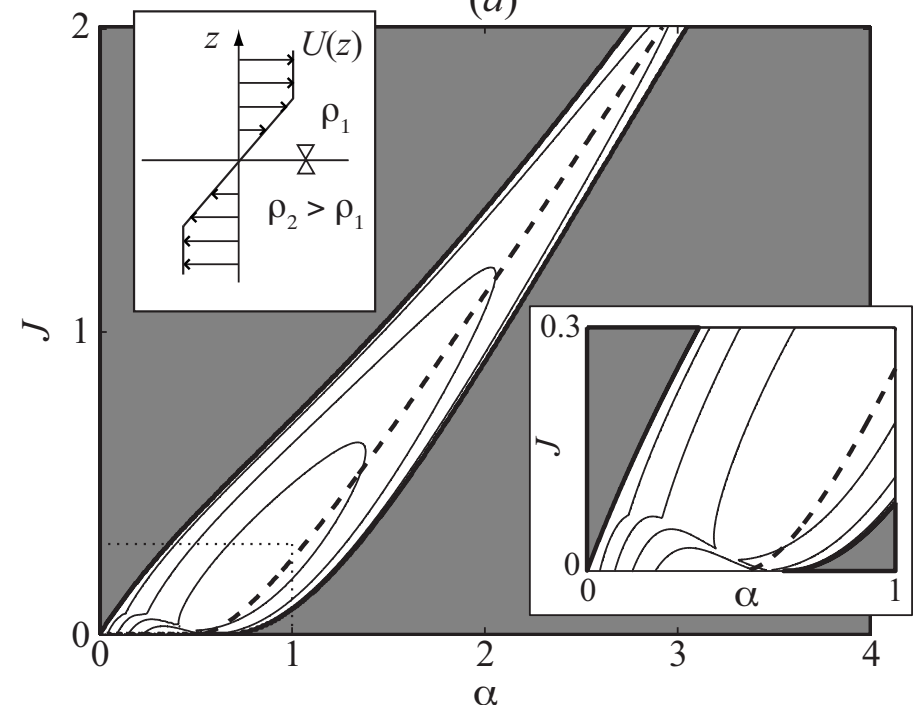

(b)

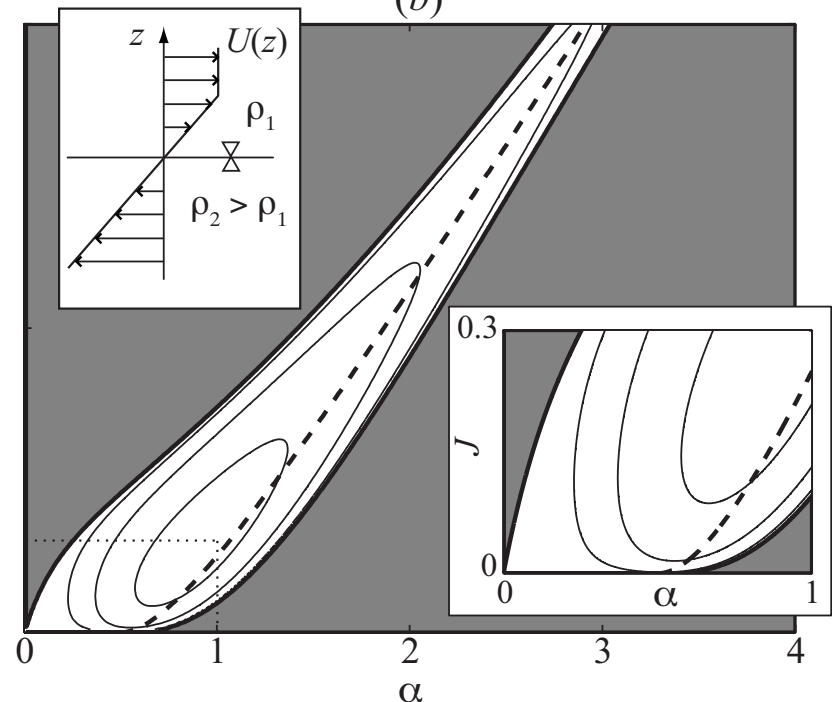

FIG. 4. Stability diagram after Baines and Mitsudera (Ref. 25) showing (a) the piecewise symmetric stratified shear layer of Holmboe (Ref. 5) and (b) the profiles of Baines and Mitsudera (Ref. 25). Contours are of growth rate with the gray shading representing regions of stability. The thick dashed line represents the resonance approximation obtained by equating the speeds of an internal wave and a vorticity wave. Profiles are shown as insets in the upper left. The dotted lines denote the boundary of the close-up regions shown as insets in the lower right.

represent the rightward-propagating $\mathrm{H}^{+}$mode, and the leftward-propagating $\mathrm{H}^{-}$mode. The close agreement between the resonance approximation and the region of instability indicates that the $\mathrm{H}$ modes are caused by an interaction between vorticity and internal wave modes. As $J$ vanishes, we approach Rayleigh's homogeneous shear layer, where instability must result from the interaction of the two vorticity modes. This was clearly illustrated by Baines and Mitsudera $^{25}$ in considering the same profiles as Holmboe ${ }^{5}$ except with the lower vorticity interface removed, shown in Fig. 4(b). By comparing the stability diagrams in Fig. 4, we see that the $\mathrm{H}$ region of instability remains largely unchanged; however, the $\mathrm{KH}$ region is completely eliminated. Since only two interfacial waves are able to interact-the upper vorticity wave and the rightward-propagating internal wave-only one type of unstable mode (the $\mathrm{H}^{+}$mode) is present. The $\mathrm{KH}$ instability is not present since it is caused by the interaction of the upper and lower vorticity modes.

In the above description, we have only concentrated on piecewise profiles. However, the same mechanisms are still believed to apply to smooth profiles. ${ }^{25}$ In this case, rather than having delta function behavior of $U^{\prime \prime}$ and $N^{2}$ at the interfaces, those functions take on smooth distributions that attain extrema in an "interfacial region." The $\mathrm{KH}$ instability is now a result of the inflection point in the $U$-profile that separates two regions of oppositely signed vorticity gradients. Likewise, the $\mathrm{H}$ instability is the interaction of a region of strong vorticity gradients $\left(U^{\prime \prime}\right)$ with a strong density gradient region $\left(N^{2}\right)$. It does not require the presence of an inflection point. Note that this is not a violation of the Rayleigh $^{3}$ inflection point theorem since this theorem applies only to homogeneous flows. Similarities between smooth profiles and piecewise profiles in terms of wave interactions have been discussed previously by Baines and Mitsudera, ${ }^{25}$ and will also be seen in the results to follow.
Stratified shear layers consisting of two density interfaces may also be susceptible to a third instability type that was first discovered by Taylor. ${ }^{30}$ The wave interactions leading to this instability were described systematically by Caulfield $^{26}$ and the first laboratory observations reported by Caulfield et $a .^{22}$ We therefore refer to these unstable modes as Taylor-Caulfield (TC) modes. The TC mode results from the interaction of two oppositely propagating waves on the density interfaces that may become phase-locked due to a background shear. ${ }^{26}$ Similar to the $\mathrm{H}$ modes, the TC modes do not require the presence of an inflection point (in fact, $U^{\prime \prime}$ may be identically zero throughout the domain). In general, the instability of a stratified shear layer may be described in terms of these three interaction types (i.e., KH, H, and TC). In Sec. III, we formulate a diagnostic in order to quantify the strength of the three types of wave interactions.

\section{FORMULATION OF A DIAGNOSTIC}

In this section, we utilize condition (ii) from Sec. II that the interacting waves must cause mutual growth in each other, to formulate a diagnostic used to interpret unstable modes of the stratified shear layer. The formulation is general, and may be applied to any profiles in which distinct interfaces can be identified. This allows for a classification of the unstable modes in terms of the $\mathrm{KH}-, \mathrm{H}-$, and $\mathrm{TC}-$ mechanisms, extending previous work by Caulfield. ${ }^{26}$

\section{A. Taylor-Goldstein equation}

We will be concerned with the small amplitude motions of an incompressible inviscid Boussinesq fluid, with perturbations taken about the basic profiles that are small enough to be well approximated by the linearized equations of motion. Following the framework of Holmboe, ${ }^{5}$ we partition the total perturbation vorticity of the flow, $q$, into a kinematic 
portion $q_{K}$, and a baroclinic portion, $q_{B}$. The kinematic vorticity is created by the vertical displacement of vorticity gradients in the $U$-profile, and is given in the linear approximation by

$$
q_{K}=-U^{\prime \prime} \eta
$$

where $\eta$ denotes the vertical displacement field. In Boussinesq fluids, baroclinic vorticity is produced by the tilting of the constant density surfaces of the $\bar{\rho}$-profile. This baroclinic production of vorticity may be written, within the linear approximation, as

$$
\frac{D q_{B}}{D t}=N^{2} \frac{\partial \eta}{\partial x},
$$

where the material derivative here and throughout the remainder of the paper, has been linearized, and is given by

$$
\frac{D}{D t} \equiv \frac{\partial}{\partial t}+U \frac{\partial}{\partial x}
$$

The total perturbation vorticity can now be written as the sum of the kinematic and baroclinic portions, viz.,

$$
q=\nabla^{2} \psi=q_{K}+q_{B} .
$$

Here we have used a stream function representation, $\psi$, of the perturbation velocity field $\mathbf{u}=(u, w)$, such that $u=\partial \psi / \partial z$ and $w=-\partial \psi / \partial x$.

Changes in the vertical displacement field may be related to the vertical velocity through the kinematic condition

$$
\frac{D \eta}{D t}=-\frac{\partial \psi}{\partial x},
$$

and allows the problem, given by Eq. (2) through Eq. (4), to be expressed in terms of a single equation for $\psi$. Perturbations can now be taken to be of the normal mode form, i.e.,

$$
\psi(x, z, t)=\hat{\psi}(z) e^{i k(x-c t)},
$$

for the stream function, where $k$ is the horizontal wave number and $c=c_{r}+i c_{i}$ is the complex phase speed. Substituting this form results in the well-known Taylor-Goldstein (TG) equation

$$
\hat{\psi}^{\prime \prime}+\left[\frac{N^{2}}{(U-c)^{2}}-\frac{U^{\prime \prime}}{U-c}-k^{2}\right] \hat{\psi}=0 .
$$

This equation, together with the condition that $\hat{\psi}$ vanishes on the boundaries (which may be taken at $z= \pm \infty$ ), describes an eigenproblem for the eigenvalue $c$, and the eigenfunction $\hat{\psi}(z)$. The flow given by the basic profiles is linearly unstable if there is an eigenvalue with $c_{i}>0$, in which case the growth rate is $\sigma=k c_{i}$.

\section{B. Partitioning into kinematic and baroclinic fields}

Once the normal modes are determined from solving the TG equation, it is possible to use this information to examine the roles that the kinematic and baroclinic fields play in the growth of the resulting instabilities. This may be accomplished by first noting that the $\psi$ due to the kinematic vortic- ity alone, may be determined directly from $\eta$. By defining this stream function field as $\psi_{K}(x, z, t)$, Eq. (4) implies that

$$
\psi=\psi_{K}+\psi_{B} \text {, and similarly } \hat{\psi}=\hat{\psi}_{K}+\hat{\psi}_{B},
$$

so that Eq. (2) may be written as

$$
\hat{\psi}_{K}^{\prime \prime}-k^{2} \hat{\psi}_{K}=-U^{\prime \prime} \hat{\eta}
$$

A similar form may be found for the baroclinic field from Eq. (3) and expressed as

$$
\hat{\psi}_{B}^{\prime \prime}-k^{2} \hat{\psi}_{B}=\frac{N^{2}}{U-c} \hat{\eta} .
$$

Since $\hat{\eta}$ and $c$ are both known from the solution to the TG equation, Eqs. (9) and (10) can be expressed in the general form

$$
L[\hat{\psi}]=f(z),
$$

where the linear operator $L=d^{2} / d z^{2}-k^{2}$, and $f(z)$ may be regarded as some known forcing function given by the right hand sides of Eqs. (9) and (10). We may now solve for $\hat{\psi}(z)$ by inverting the operator $L$ by the relation

$$
\hat{\psi}=\int_{D} G(s, z) f(s) d s,
$$

where $D$ is the domain, and $G(s, z)$ is the appropriate Green's function for $L$, which depends on the boundary conditions. In this case it is given by

$$
G(s, z)=\frac{\cosh k\left(z_{u}+z_{l}-z-s\right)-\cosh k\left(z_{u}-z_{l}-|z-s|\right)}{2 k \sinh k\left(z_{u}-z_{l}\right)},
$$

for domains with upper and lower boundaries at $z_{u}$ and $z_{l}$, respectively. When the domain is unbounded then this reduces to $G(s, z)=-e^{-k|z-s|} / 2 k$.

From Eq. (12), it is possible to partition the $\hat{\psi}$ into kinematic and baroclinic effects. The contribution of each field to the growth rate and phase speed of the normal mode disturbance can now be explicitly solved for by rearranging the kinematic condition (5) and substituting the normal mode form (6) to give

$$
c_{r}=U+\operatorname{Re}\left(\frac{\hat{\psi}_{K}+\hat{\psi}_{B}}{\hat{\eta}}\right),
$$

and

$$
\sigma=k \operatorname{Im}\left(\frac{\hat{\psi}_{K}+\hat{\psi}_{B}}{\hat{\eta}}\right) .
$$

The relation for $\sigma$ in Eq. (15) will be used throughout the remainder of the paper to assess the contributions of the kinematic and baroclinic fields in the growth of unstable modes. 


\section{Piecewise profiles}

We now apply these general relations to the piecewise profiles of Fig. 1, leaving the formulation in terms of an arbitrary number of multiple interfaces to Appendix B. Since these profiles exhibit delta function behavior at the interface locations, we may write

$$
U^{\prime \prime}(z)=\Delta q_{1} \delta\left(z-z_{1}\right)+\Delta q_{2} \delta\left(z-z_{2}\right)
$$

and

$$
N^{2}(z)=g^{\prime} \delta(z+d)
$$

where the subscript 1 and 2 refer to the upper and lower vorticity interfaces, respectively. Substituting the above into Eqs. (9) and (10) and utilizing Eq. (12) allows us to determine the stream function associated with each interface given the interfacial displacement $(\hat{\eta})$, as found by solving the TG equation. We can then write these stream functions as $\hat{\psi}_{K 1}, \hat{\psi}_{K 2}$, and $\hat{\psi}_{B}$, for the upper vorticity, lower vorticity, and density interface, respectively. It is now a simple matter to use Eq. (15) to express the total growth rate ( $\sigma$, obtained by the solution to the TG equation) at each interface as a sum of the growth rate contributions of all other interfaces. For example, we may choose the upper vorticity interface (denoted by the $K 1$ superscript) and write

$$
\begin{aligned}
\sigma & =k \operatorname{Im}\left[\frac{\hat{\psi}_{K 1}\left(z_{1}\right)+\hat{\psi}_{K 2}\left(z_{1}\right)+\hat{\psi}_{B}\left(z_{1}\right)}{\hat{\eta}\left(z_{1}\right)}\right], \\
& =\sigma_{K 1}^{K 1}+\sigma_{K 2}^{K 1}+\sigma_{B}^{K 1} .
\end{aligned}
$$

Each of the growth rate terms on the right hand side of Eq. (19) will be referred to as a partial growth rate. In a similar fashion, we can repeat this for the other $K 2$ and $B$ interfaces present. This leads to three sums as in Eq. (19) above, one for each interface, and a total of nine different partial growth rates. However, it is not possible for a vorticity interface to cause growth in itself (i.e., $\sigma_{K j}^{K j}=0$ for any vorticity interface $j$ ), and so the number of terms in the partial growth rate sums are reduced. This "self-interaction" is not possible since $\hat{\eta}$ and $\hat{\psi}_{K}$ must always have a phase difference of 0 or $\pi$ radians, as can be seen from Eqs. (9) and (12).

\section{Smooth profiles}

The same partial growth rate diagnostic is now developed for the continuous distributions of $U^{\prime \prime}$ and $N^{2}$, with the generalization to an arbitrary number of interfaces described in Appendix B. This is done by defining three interfacial regions where either $U^{\prime \prime}$ or $N^{2}$ reaches an extrema (see Fig. 5). The vorticity interface regions in this case extend from the upper and lower boundaries to the inflection point at $z=0$. Since the $N^{2}$ profile exhibits only a single maximum at $z=-d$, the density interface region is the entire domain, $D$. The $\hat{\psi}_{K, B}$ can then be defined for each interface using Eq. (12) as

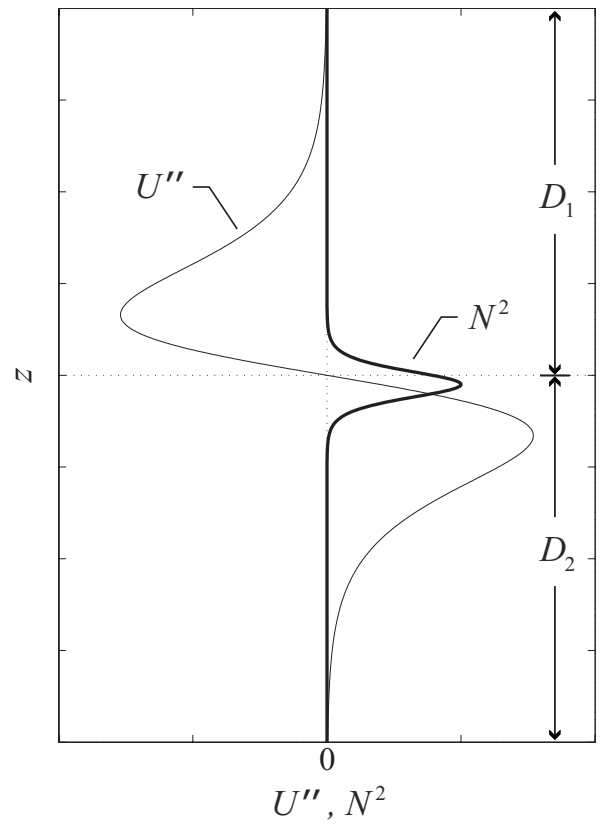

FIG. 5. Plots of $U^{\prime \prime}(z)$ and $N^{2}(z)$ for the smooth profiles shown in Fig. 1. The position of the upper and lower interfacial vorticity regions are labeled as $D_{1,2}$, respectively.

$$
\hat{\psi}_{K 1,2}(z)=-\int_{D_{1,2}} G(s, z) U^{\prime \prime}(s) \hat{\eta}(s) d s,
$$

in the case of the vorticity interfaces, with $D_{1}=[0,+\infty)$, $D_{2}=(-\infty, 0]$, and

$$
\hat{\psi}_{B}(z)=\int_{D} G(s, z) \frac{N^{2}(s)}{U(s)-c} \hat{\eta}(s) d s,
$$

for the density interface.

Now that the stream function associated with each interface is known, we may once again use Eq. (15) and $\hat{\eta}$ to calculate the partial growth rates. The difficulty with this is that we do not know at what precise vertical location the interface is located at; in smooth profiles we are only able to identify interfacial regions. It is intuitively clear that the interface should be concentrated near the extrema of $U^{\prime \prime}$ or $N^{2}$. We therefore use these profiles as weight functions in an integrated version of (15). Using the upper vorticity interface as an example, we multiply both sides by $U^{\prime \prime}$ and integrate over the interfacial region $D_{1}$, represented by \langle\rangle$_{D_{1}}$ to give

$$
\begin{aligned}
\sigma & =\frac{\left\langle U^{\prime \prime} \pi_{K 1}\right\rangle_{D_{1}}}{\left\langle U^{\prime \prime}\right\rangle_{D_{1}}}+\frac{\left\langle U^{\prime \prime} \pi_{K 2}\right\rangle_{D_{1}}}{\left\langle U^{\prime \prime}\right\rangle_{D_{1}}}+\frac{\left\langle U^{\prime \prime} \pi_{B}\right\rangle_{D_{1}}}{\left\langle U^{\prime \prime}\right\rangle_{D_{1}}}, \\
& =\sigma_{K 1}^{K 1}+\sigma_{K 1}^{K 2}+\sigma_{K 1}^{B},
\end{aligned}
$$

after dividing by both sides by $\left\langle U^{\prime \prime}\right\rangle_{D_{1}}$. Here we have defined $\pi_{j}(z)=k \operatorname{Im}\left(\hat{\psi}_{j} / \hat{\eta}\right)$, where $j$ represents one of the three $\{K 1, K 2, B\}$ interfaces of interest. This gives us a direct analogue to Eq. (19) for smooth profiles (although the choice of $U^{\prime \prime}$ or $N^{2}$ as weight functions is a little arbitrary, we have verified that other selections provide qualitatively similar results). Partial growth rate sums as in Eq. (23) can also be developed for the remaining two interfaces by choosing the 
(a) $\sigma$

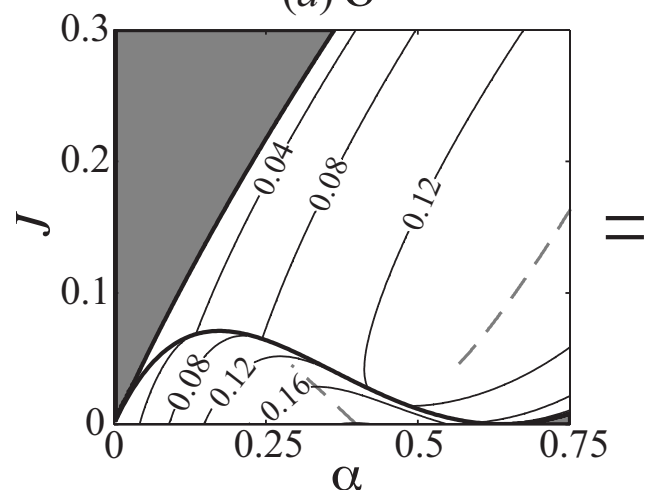

(b) $\sigma$

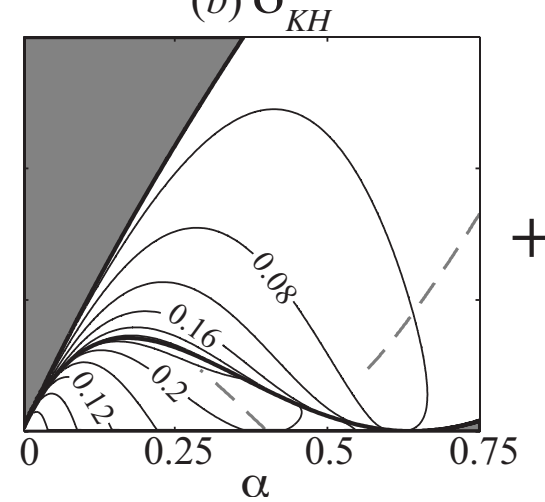

(c) $\sigma_{H}$

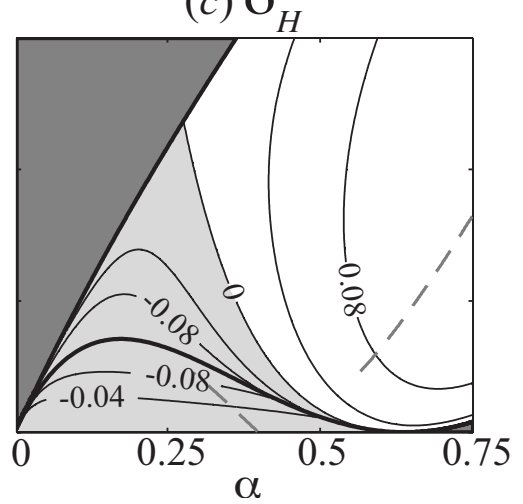

FIG. 6. Partial growth rates for the symmetric case using piecewise profiles. Contours of $\sigma$ are shown in (a), which is given as the sum of $\sigma_{\mathrm{KH}}$ in (b), and $\sigma_{\mathrm{H}}$ in (c). The thick solid line denotes the stability boundary and transition from stationary $\left(c_{r}=0\right)$ to propagating $\left(c_{r} \neq 0\right)$ modes. Dark gray shading indicates a region of stable modes, whereas light gray shading denotes regions where the partial growth takes negative values. The position of the wave number of maximum growth rate is indicated by the gray dashed line.

appropriate weight function $\left(N^{2}\right.$ in the case of the density interface) and region of integration. It should be noted that for smooth profiles we do not necessarily have $\sigma_{K j}^{K j}=0$, as for piecewise profiles. This self-interaction is possible since phase differences should be expected between $\hat{\psi}_{K}$ and $\hat{\eta}$ within the interfacial region.

\section{RESULTS}

We now apply the partial growth rate formulation for piecewise and smooth profiles to the stratified shear layers in Fig. 1. The symmetric case is examined first, followed by the asymmetric case where we let $a=0.5$. This particular value for $a$ was chosen to match the asymmetry that Lawrence et $a l .{ }^{20}$ observed in their laboratory experiments. In the case of smooth profiles, we will take

$$
U(z)=\frac{\Delta U}{2} \tanh \left(\frac{2 z}{h}\right),
$$

and

$$
\bar{\rho}(z)=\rho_{0}-\frac{\Delta \rho}{2} \tanh \left(\frac{2 R z}{h}+a\right),
$$

and examine a single value of the interfacial thickness ratio $R=5$, which is large enough to permit a significant region of unstable propagating modes when $a=0$.

The profiles consist of two vorticity interfaces and a single density interface. Therefore, the TC mode that results from the interaction of two density interfaces can immediately be disregarded. The partial growth rate diagnostics in Eq. (19) or Eq. (23) reduces to a sum of three terms for each interface. However, we will limit our attention to only the rightward-propagating $\mathrm{H}^{+}$mode, the $\mathrm{KH}$ mode, as well as the dominant asymmetric mode. In doing so, it will suffice to apply the partial growth rate diagnostic only to the upper vorticity interface, since each unstable mode will consist of mutual growth between the upper vorticity interface, and one of the lower vorticity or density interfaces. The diagnostic equation then becomes

$$
\sigma=\sigma_{\mathrm{KH}}+\sigma_{\mathrm{H}}+\sigma_{\text {self }},
$$

where the terms on the right hand side represent the partial growth rate due to the interaction between the upper vorticity interface and (from left to right) the lower vorticity, density, and upper vorticity interfaces. These terms are identified with $\mathrm{KH}, \mathrm{H}$, and self-interaction components, respectively.

\section{A. Symmetric profiles}

Beginning with the stability properties of the piecewise symmetric stratified shear layer, we plot the partial growth rates from Eq. (26) in Fig. 6. Recall that a vorticity interface cannot interact with itself; therefore, for all the piecewise results $\sigma_{\text {self }}=0$ and only $\sigma_{\mathrm{KH}}$ and $\sigma_{\mathrm{H}}$ are required in the sum, i.e., the normal mode growth rate can be expressed as a $\mathrm{KH}$ and $\mathrm{H}$ component according to Eq. (26).

In the region of stationary $\left(c_{r}=0\right)$ instability, growth is due entirely to $\sigma_{\mathrm{KH}}$ [Fig. 6(b)], while the density interface acts as a stabilizing influence, indicated by the negative values of $\sigma_{\mathrm{H}}$ [Fig. 6(c)]. In addition, the asymptotic result of an unstable $\mathrm{H}$ mode consisting predominantly of a $\sigma_{\mathrm{H}}$ component is recovered for large $\alpha$ (and $J$ ), as expected. As $J$ increases, the $\mathrm{KH}$ contribution, $\sigma_{\mathrm{KH}}$, first increases to a maximum near the transition from stationary to propagating modes, and then slowly declines towards zero. This behavior indicates that the interaction of vorticity interfaces, normally associated with the stationary $\mathrm{KH}$ mode, does contribute to the growth of the propagating modes, particularly near the transition between the two. In other words, the KH mechanism exerts a significant influence in the region of propagating modes.

This result should not be surprising if we consider, once again, the stability diagrams of Fig. 4. The profiles of Baines and Mitsudera ${ }^{25}$ [Fig. 4(b)] show a purely H-type growth mechanism since no lower vorticity interface is present. When this lower vorticity interface is introduced in Fig. 4(a), the $\mathrm{KH}$ region is produced in the stability diagram. However, the growth rates in the propagating modes are also altered by the addition of the lower vorticity interface, indicating that the $\mathrm{KH}$ mechanism alters the growth rates of the propagating 

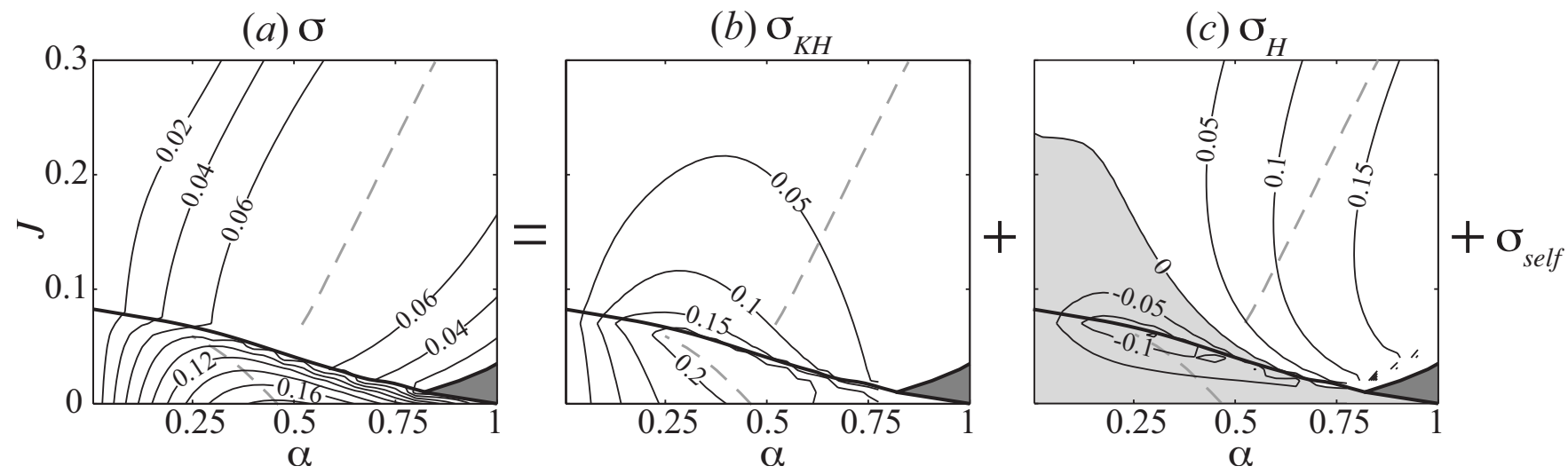

FIG. 7. Partial growth rates for the symmetric case using smooth profiles. All notation as in Fig. 6 except $\sigma_{\text {self }}$ (not shown) must be included to complete the sum.

modes. The KH contribution to the total growth rate of the propagating modes is greatest close to the transition, and it should be noted that the neglect of the lower vorticity interface does not significantly affect the stability properties of the propagating modes at large $J$ and $\alpha$.

The same general behavior of the piecewise profiles is also seen in the partial growth rates of the smooth profiles shown in Fig. 7. Note that because the TG equation is solved numerically on a finite domain for the smooth profiles (the domain height was here taken to be $10 h$ ), the resulting growth rates in Fig. 7(a) exhibit certain differences with their counterparts for the piecewise profiles in Fig. 6(a), and, in particular, the low- $\alpha$ modes become unstable. ${ }^{6}$ In addition, when examining smooth profiles, $\sigma_{\text {self }}$ must be included in order to complete the sum in Eq. (26). Although $\sigma_{\text {self }}$ can be a large term, it is always found to be a negative (stabilizing) influence, and does not significantly affect the interpretation of the dominant instability mechanism. For this reason we do not show the details of $\sigma_{\text {self }}$ in the results.

Just as in the piecewise results in Fig. 6, the continuous profiles show that the stationary modes are dominated by the $\mathrm{KH}$ component of the partial growth rates. This persists into the propagating region, particularly at small $\alpha$, where $\sigma_{\mathrm{H}}$ continues to be negative. As $J$ is increased, the $\mathrm{H}$ interaction between shear layer vorticity and the density interface becomes the dominant mechanism of instability growth. We can again conclude that a significant portion of the propagating modes experience a $\mathrm{KH}$-type mechanism of inflection point growth near the phase speed transition.

Since finite amplitude instabilities in stratified shear layers have generally been found to occur at the wave number where the growth rate is a maximum, denoted by $\alpha_{\max }$, it is these disturbances which we should expect to be the most relevant. ${ }^{31}$ It is therefore of interest to compare the relative contributions of $\sigma_{\mathrm{KH}}$ and $\sigma_{\mathrm{H}}$ to the growth of the instability along the wave number of maximum growth curve. This is shown in Fig. 8 where the partial growth rates are plotted as a function of $J$ along the $\alpha_{\max }$-curve. Note that since $\alpha_{\max }$ changes discontinuously across the transition from stationary to propagating modes, Fig. 8 shows that $\sigma_{\mathrm{KH}}$ and $\sigma_{\mathrm{H}}$ are also discontinuous there. There are two locations of particular interest in these plots: the transition from stationary to propa- gating modes (vertical gray line), and the point at which $\sigma_{\mathrm{KH}}=\sigma_{\mathrm{H}}$ (vertical dashed gray line). The first location denotes the commonly accepted transition between $\mathrm{KH}$ and $\mathrm{H}$ modes based on phase speed considerations, and the second shows our diagnostic describing the predominance of either the $\mathrm{KH}-$ or H-type growth mechanisms. In the case of the piecewise profiles of Fig. 8(a), these two locations are coincident. However, the smooth profiles show that H-type growth does not predominate until $J$ has surpassed the transition to propagating modes. The partial growth rate diagnostic appears to provide a good description of the nonlinear dynamics for the few simulations performed, as shown in Figs. 8(c)-8(f).
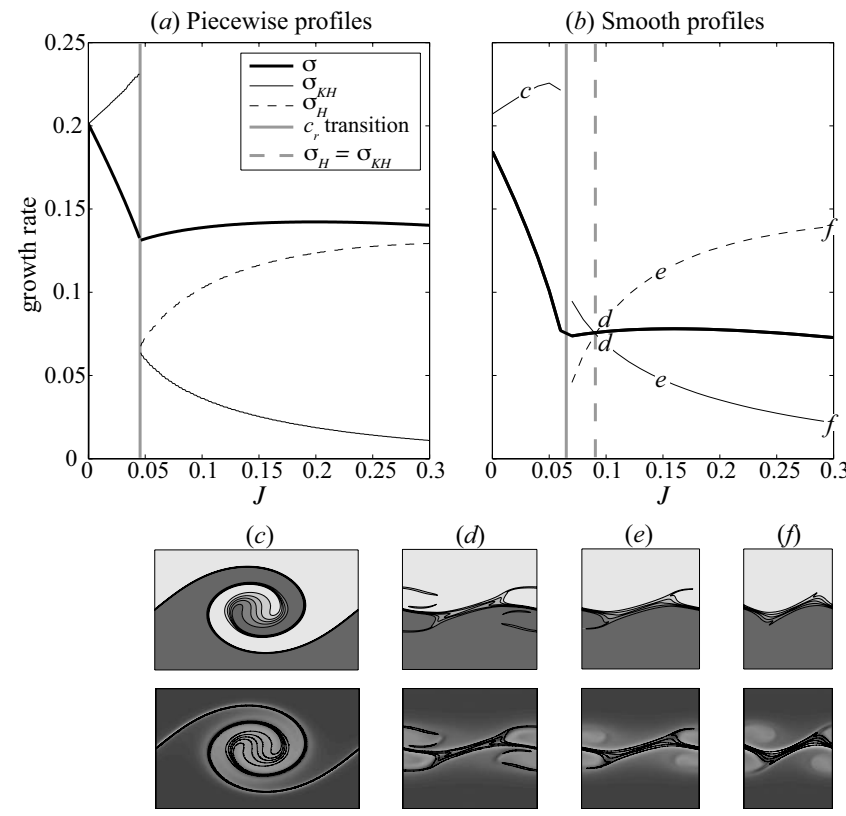

FIG. 8. Partial growth rates for the symmetric case along $\alpha_{\max }$. Piecewise results are shown in (a), while (b) gives the results for smooth profiles along with the numerical results illustrating the nonlinear form of the instabilities [(c)-(f)]. The transition between stationary and propagating modes is given by the vertical gray line (with $c_{r}=0$ for small $J$ and $c_{r} \neq 0$ at larger $J$ ). The dashed gray line denotes where $\sigma_{\mathrm{KH}}=\sigma_{\mathrm{H}}$. Negative partial growth rates are not shown. 
(a) $\sigma$

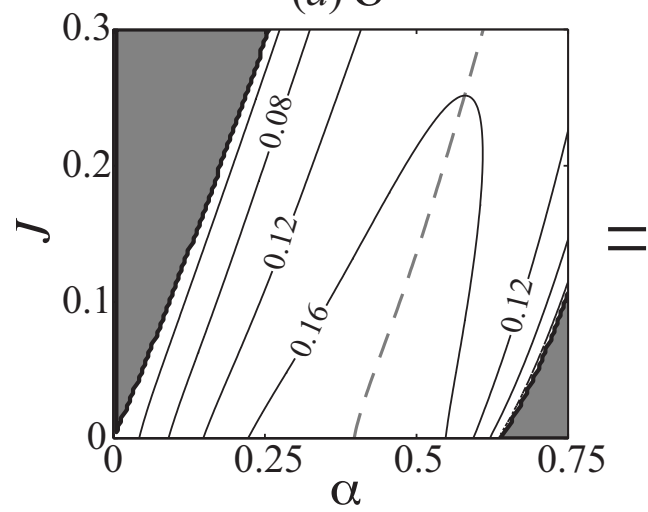

(b) $\sigma$

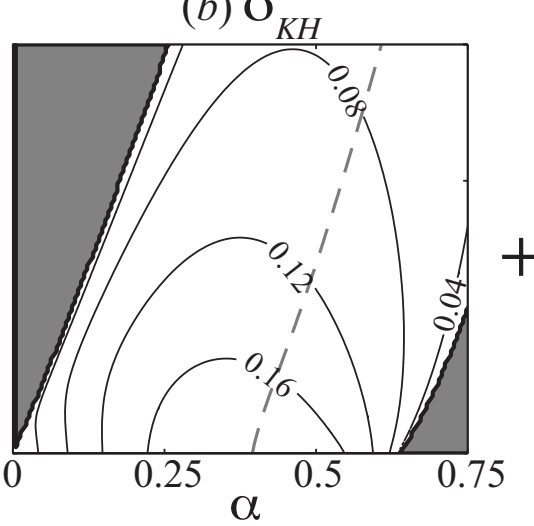

(c) $\sigma_{H}$

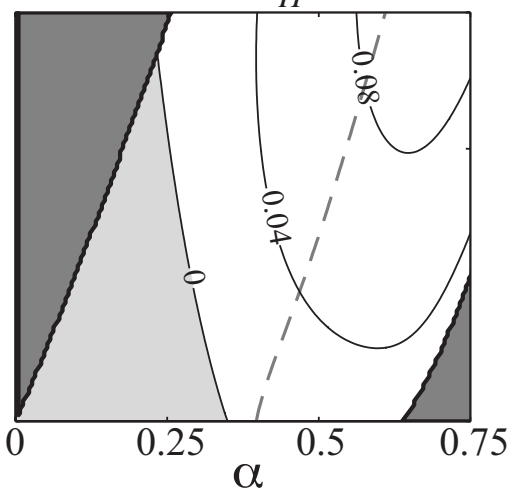

FIG. 9. Partial growth rates for the dominant mode of the asymmetric $(a=0.5)$ case using piecewise profiles. Details as in Fig. 6 .

\section{B. Asymmetric profiles}

Whereas the symmetric profiles show a distinct transition from stationary to propagating instabilities, there is no such transition in the case of asymmetric profiles. Instead, two unstable propagating $\left(c_{r} \neq 0\right)$ modes, each traveling in opposite directions with respect to the mean flow, are present for all $J>0$. However, we note that as $J \rightarrow 0$ we recover Rayleigh's homogeneous shear layer instability, and so KH behavior is expected at small $J$. Also, for large $J$ (and large $\alpha$ ) it can be seen that both instability regions are in alignment with the resonance condition between the vorticity interfaces and the interfacial waves on the density interface (obtained by equating the phase speeds of each pair of waves, see Sec. II). We therefore expect that the unstable modes are of H-type at large $J$, and some sort of transition between the two linear growth mechanisms must take place.

This behavior is reflected in the partial growth rates of the piecewise profiles shown in Fig. 9. Only the dominant mode consisting of larger growth rates is plotted since it has most often been found to control the development of the instabilities into the nonlinear regime. ${ }^{20,23}$ Figure 9 shows that both $\sigma_{\mathrm{KH}}$ and $\sigma_{\mathrm{H}}$ are positive over the majority of the $\alpha J$-plane where the unstable mode is present. Since $\sigma_{\mathrm{KH}}$ is a decreasing function of $J$, and $\sigma_{\mathrm{H}}$ is an increasing function of $J$, we expect a gradual transition from $\mathrm{KH}$ - to $\mathrm{H}$-type modes. In other words both $\mathrm{KH}$ and $\mathrm{H}$ growth mechanisms are present throughout the majority of the unstable region, and they replace one another in a gradual fashion as $J$ is increased.

The resulting plots for smooth profiles (Fig. 10) show qualitatively similar results. Some difficulty was encountered in the numerical solution of the TG equation for smooth profiles near the stability boundary due to the near-singular behavior of the solutions at the critical level (see Alexakis ${ }^{32}$ for further discussion). Evidence of this can be seen in Fig. $10(\mathrm{c})$, where the contouring of $\sigma_{\mathrm{H}}$ is a little irregular; however, we do not expect this to have significantly effected the results.

Focusing on the most amplified wave number, Fig. 11 shows a gradual transition from a growth dominated by $\sigma_{\mathrm{KH}}$ to one where $\sigma_{\mathrm{H}}$ becomes the stronger influence, as $J$ is increased. This is in accord with previous observations of the nonlinear behavior of the instabilities, ${ }^{20,24}$ and can also be seen in the results from the numerical simulations [Figs. 11(c)-11(f)]. A KH-like behavior can be seen in the instability for $J=0.03$ and 0.10 in Figs. 11(c) and 11(d), where significant overturning is present in the density field, and this is to be expected with a larger value of $\sigma_{\mathrm{KH}}$. By $J=0.30$, in Fig. 11(f), we have a nonlinear form of the instability that is more akin to the $\mathrm{H}$ instability, resembling a cusplike wave. There is only a gradual transition visible in the nonlinear forms of the instabilities, and they generally appear as a mix of both (a) $\sigma$

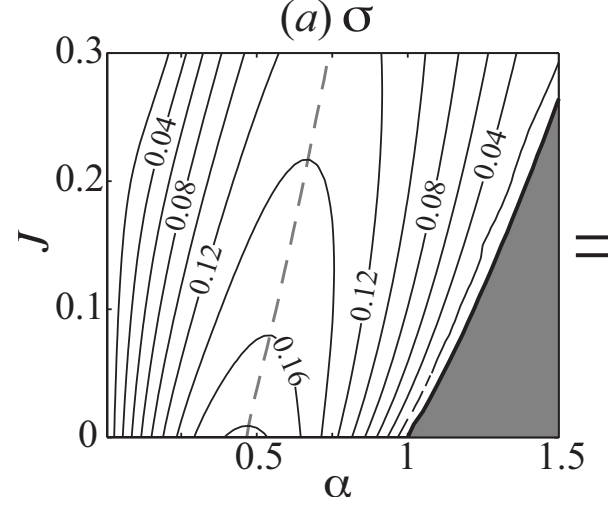

(b) $\sigma_{K}$

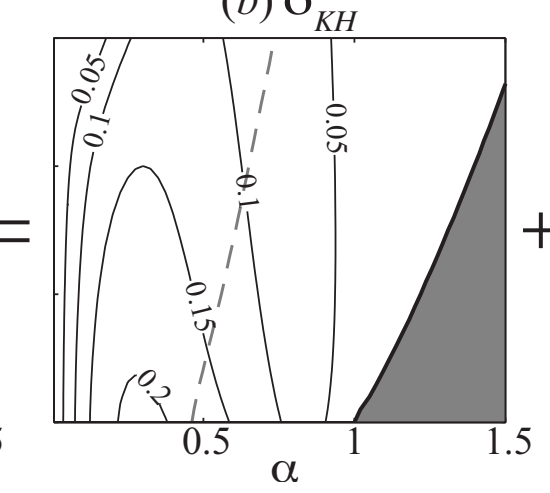

(c) $\sigma_{H}$

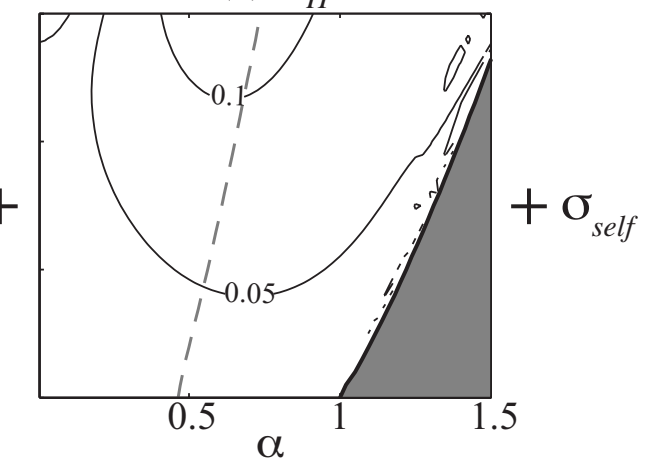

FIG. 10. Partial growth rates for the asymmetric $(a=0.5)$ case using smooth profiles. Details as in Fig. 7. 

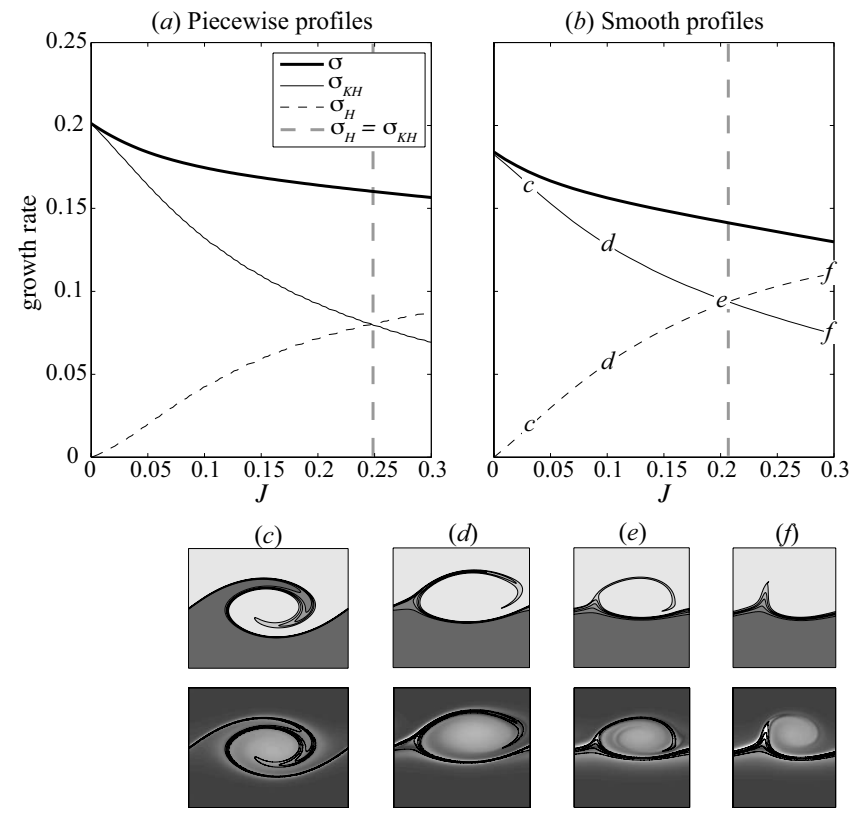

FIG. 11. Partial growth rates for the asymmetric case along the wave number of maximum growth. Details as in Fig. 8.

types-also to be expected from the partial growth rate predictions. A result that can be seen directly from Fig. 11 is that the asymmetry extends the $\mathrm{KH}$ mechanism to larger values of $J$ than in the symmetric case.

\section{APPLICATION TO FIELD PROFILES}

We now give an example of how to apply the diagnostic to $U$ and $\bar{\rho}$ profiles collected from the field. The profiles shown in Fig. 12 were measured in the Fraser River estuary, which have been smoothed and filtered using the methods described in Tedford et al. ${ }^{18}$ Both of the $U$ and $\bar{\rho}$ profiles can be seen to consist of a number of "layers," which lead to many possible interactions between interfaces. These interfaces can be identified by extrema in the profiles of $U^{\prime \prime}$ and $N^{2}$ shown in Figs. 12(a) and 12(b), respectively. The presence of multiple inflection points in $U$, and a number of maxima in $N^{2}$ indicate that any of the three instability types (KH, H, and TC) mentioned in Sec. II may be present. A linear stability analysis of these profiles predicts an unstable mode with a maximum growth rate of $\sigma=0.024 \mathrm{~s}^{-1}$ at a wave number of $k=0.61 \mathrm{~m}^{-1}$ and a displacement eigenfunction amplitude $|\hat{\eta}|$, shown in Fig. 12(c). These predictions of linear theory were found in Tedford et $a l^{18}$ to be in good agreement with observations recorded by an echosounder [Fig. 12(d)], both in the wave number $k$, and the vertical location given by the maximum of $|\hat{\eta}|$.

We now determine which interfacial wave interactions are responsible for generating the instabilities seen in the echosounding of Fig. 12(d). Due to the many interfaces that are present in the profiles, we shall simplify the analysis by noting that the dominant vertical location of the perturbations is concentrated near the peak of $|\hat{\eta}|$ at $z=10.9 \mathrm{~m}$. This suggests that the instability is due only to the influence of the interfaces nearest this level. These consist of the two uppermost vorticity interfaces, with extrema at $z \approx 11.5$ and $10 \mathrm{~m}$, as well as the density interface at $z \approx 10.5 \mathrm{~m}$. Assuming this to be the case, we modify the observed profiles so that $U^{\prime \prime}$ and $N^{2}$ vanish below, with $U^{\prime}$ remaining constant. The modified profiles will be denoted by an asterisk subscript (e.g., $\left.U_{*}\right)$, and are shown in Fig. 12. Performing a linear stability analysis on the modified profiles yields only a $1 \%$ change in the peak growth rate, and negligible changes in $k_{\max }$ and $|\hat{\eta}|$ [see Fig. 12(c)]. We conclude that our choice of modified profiles is justified by these negligible changes in the properties of the unstable mode (the mathematical reason being the decay of the Green's function (13) away from the interfacial regions). We have also confirmed that the neglected interfaces in the original $U$ and $N^{2}$ profiles have negligible partial growth rate contributions.

The $U_{*}$ and $N_{*}^{2}$ profiles are of similar form to the asymmetric stratified shear layer examined in Sec. IV with the exception that the two vorticity interfaces are of different strength, and in the location of the vertical boundaries. Once again we identify a $\sigma_{\mathrm{KH}}, \sigma_{\mathrm{H}}$, and $\sigma_{\text {self }}$ associated with the partial growth rates of the upper vorticity interface with the lower vorticity, density, and upper vorticity interfaces, re-
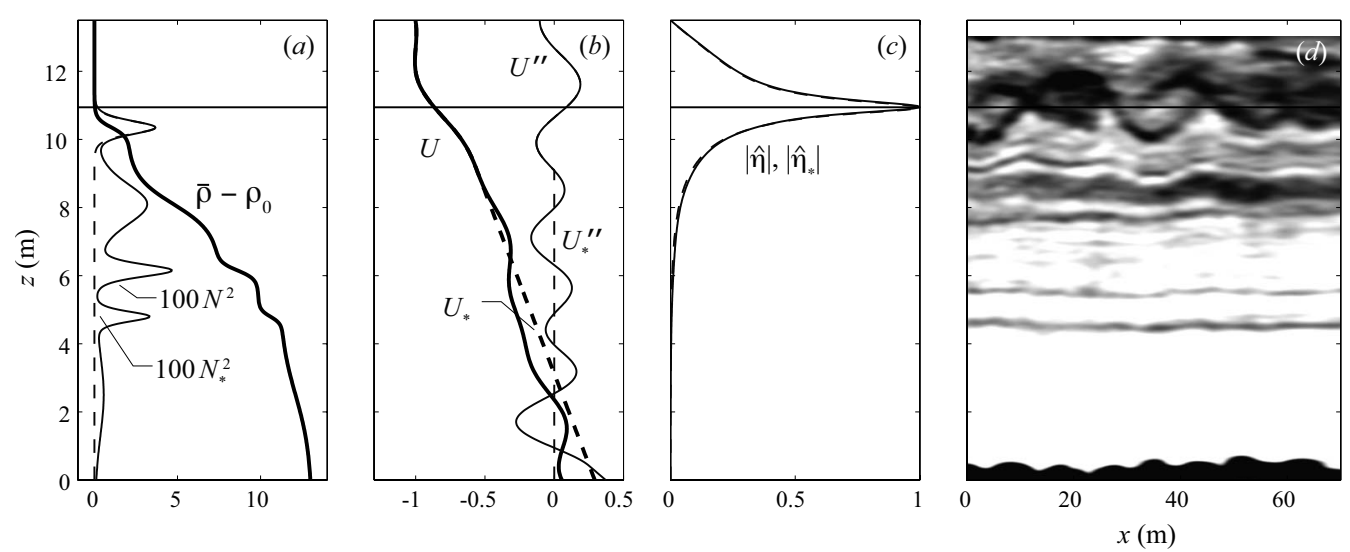

FIG. 12. Profiles taken from field measurements in the Fraser River estuary [see Tedford et al. (Ref. 18) for details]. In [(a)-(c)], the original and modified profiles are denoted by solid and dashed curves, respectively. The density structure is plotted in (a) and the velocity structure in (b). The normalized amplitude of the displacement eigenfunction, is shown in (c) with the solid horizontal line indicating the vertical location of the peak displacement. An echosounding image taken at a time close to the profiles [(a) and (b)] is shown in (d), where instability can be seen near the peak in $|\hat{\eta}|$. 
spectively. Applying the diagnostic formulated in Sec. III results in $\left\{\sigma_{\mathrm{KH}}, \sigma_{\mathrm{H}}, \sigma_{\text {self }}\right\}=\{0.011,0.023,-0.010\} \mathrm{s}^{-1}$. This leads us to conclude that the instabilities observed in Fig. 12 are primarily of the H-type, and appears to be in agreement with the wavelike features observed in the echosounding image in Fig. 12(d) (though the interpretation of density structure from echosounding images is imprecise, see Tedford et al. ${ }^{18}$ and references therein). These results also add further quantitative evidence to support the observations of Tedford et al. ${ }^{18}$ to have identified instabilities primarily of the H-type. To our knowledge this would be the first time that H-type instabilities have been identified in the field.

\section{DISCUSSION AND CONCLUSIONS}

Based on the wave interaction view of stratified shear instability, ${ }^{5,25,26}$ a diagnostic has been developed to interpret the wave interactions in stratified shear layers that lead to the growth of unstable modes. It can be viewed as an extension of the resonance and large- $\alpha$ approximations used by Caulfield, ${ }^{26}$ Caulfield et $a l .{ }^{22}$ and Baines and Mitsudera, ${ }^{25}$ to allow for a general classification of stratified shear instabilities in terms of the $\mathrm{KH}-, \mathrm{H}-$ and $\mathrm{TC}-$-mechanisms. These types refer to vorticity-vorticity, vorticity-internal wave, and internal wave-internal wave interactions, respectively. The diagnostic has the advantage of quantifying wave growth in the entire $\alpha J$-plane, as well as being applicable to smooth profiles.

The diagnostic is applied first to the symmetric and asymmetric stratified shear layers. An interesting result of the analysis was the observation that the $\mathrm{KH}$ mechanism contributed significantly to the propagating modes that are normally classified as H-type. The asymmetric stratified shear layer, whose region of instability consists entirely of propagating modes, was generally found to be composed of a mix of both $\mathrm{KH}-$ and H-type growth mechanisms. This highlights the fact that in general stratified shear flows a strict classification into one mode type or another is not always possible, and that an alternative is to quantify the mechanisms that are present. The diagnostic suggests that the KH mechanism is found at larger values of $J$ in the asymmetric case, and appears to be in qualitative agreement with results from the numerical simulations presented here, as well as those of Carpenter et al. ${ }^{14}$ which cover a range of asymmetries.

A potential drawback of the method is the large number of wave interactions that must be accounted for in profiles with multiple interfaces. In the field profiles examined from the Fraser River estuary, the number of observed interfaces would produce an unwieldy number of interactions that must be accounted for. This issue was dealt with by using a knowledge of the unstable mode to simplify the profiles without significantly affecting the stability properties. This reduced the analysis to that of the stratified shear layers examined earlier. However, this simplification may not always be possible and there may arise cases in which the instability involves the interaction of numerous interfaces.

Extending the use of the diagnostic to smooth profiles allows for the direct interpretation of wave interactions in geophysically relevant profiles measured in the field. These profiles invariably display some type of asymmetry, and the resulting modes of instability are likely to be of a "mixed" or "hybrid" type, i.e., they may involve two or more interaction types as is found in this study. This behavior has been noted in numerous cases exhibiting asymmetry in a more general sense, for example, the non-Boussinesq flow of Umurhan and Heifetz, ${ }^{33}$ and the spatially developing flows of Pawlack and $\mathrm{Armi}^{21}$ and Ortiz et al. ${ }^{34}$ in addition to the vertically offset profiles considered in the present study. In these mixed mode circumstances, the partial growth rate diagnostic may provide a useful tool in assessing the characteristics of the instability so that predictions can be made regarding the nonlinear dynamics.

\section{ACKNOWLEDGMENTS}

The authors would like to thank the two referees for valuable comments, Ted Tedford for helpful discussions on this work and for his analysis of the Fraser River data. T. Tedford, R. Pawlowicz, R. Pieters, and M. Rhode also contributed to the collection of this data. We would also like to thank M. Rahmani for help with the preparation of the figures, and W. Smyth for his contribution to an earlier version of this paper which was presented at the Sixth International Symposium on Stratified Flows on 11-14 December 2006 in Perth, Western Australia. Simulations were performed on WestGrid.

\section{APPENDIX A: DESCRIPTION OF THE NUMERICAL SIMULATIONS}

Results of the DNS shown in Figs. 2, 3, 8, and 11, were performed using the code described by Winters et al. ${ }^{35}$ The DNS were two dimensional with periodic boundary conditions in the horizontal and free-slip conditions on the vertical boundaries. In each case, a single wavelength of the instability was simulated by choosing the horizontal domain length $L_{x}=\lambda_{\mathrm{FGM}}$, the wavelength of the fastest growing mode predicted from linear theory. The vertical domain height $L_{y}=10 \mathrm{~h}$, which has been found sufficiently large to agree well with the unbounded results from linear theory. The velocity and density fields were perturbed initially with the eigenfunctions so that they can develop relatively quickly into finite amplitude waves.

Although the stability analysis and diagnostic formulation applies in the inviscid, nondiffusive case only, it is necessary to choose finite kinematic viscosity, $\nu$, and diffusivity of the stratifying agent, $\kappa$, in the simulations so that they remain well resolved. The relative size of these are measured by the Reynold's number $\operatorname{Re}=\Delta U h / \nu$, and the Prandtl number $\operatorname{Pr}=\nu / \kappa$, which were set to values of 1200 and 25 , respectively. Results from a linear stability analysis incorporating the viscous and diffusive effects at these values of Re and Pr show only small differences in growth rate to the inviscid, nondiffusive case near $\alpha_{\max }$, where the DNS were performed. For this reason we expect the DNS results to apply well to the inviscid, nondiffusive problem, justifying a comparison between the finite amplitude instabilities and the results of linear theory. 


\section{APPENDIX B: GENERAL FORMULATION OF THE PARTIAL GROWTH RATE DIAGNOSTIC}

We now derive the partial growth rate diagnostic for multiple vorticity and density interfaces for piecewise profiles. The vorticity and density gradients $\left(U^{\prime \prime}\right.$ and $\left.N^{2}\right)$ exhibit delta function behavior at a number of discrete interface locations, allowing us to write

$$
U^{\prime \prime}(z)=\sum_{j=1}^{n} \Delta q_{j} \delta\left(z-z_{j}\right)
$$

and

$$
N^{2}(z)=\sum_{\ell=1}^{m} g_{\ell}^{\prime} \delta\left(z-z_{\ell}\right)
$$

where we are considering general profiles consisting of $n$ vorticity interfaces with jumps $\Delta q_{j}$, and $m$ density interfaces with jumps $g_{\ell}^{\prime}$, at the vertical locations $z_{j}$ and $z_{\ell}$, respectively.

When the delta-function forms in Eqs. (B1) and (B2) are substituted into Eq. (12), the integrals for $\hat{\psi}_{K}$ and $\hat{\psi}_{B}$ reduced to sums, where each term represents the contribution of a particular interface. If we now choose an interface of interest, the $p$ th interface say, and apply Eq. (15), we are able to break the total growth rate of the normal mode $\sigma$, into the individual contributions of each interfacial wave. This allows us to write

$$
\sigma=\sum_{j=1}^{n} \sigma_{K j}^{p}+\sum_{\ell=1}^{m} \sigma_{B \ell}^{p},
$$

where each term of the sums represents a partial growth rate, and are given explicitly by

$$
\sigma_{K j}^{p}=-k \operatorname{Im}\left\{\Delta q_{j} G\left(z_{j}, z_{p}\right) \frac{\hat{\eta}\left(z_{j}\right)}{\hat{\eta}\left(z_{p}\right)}\right\},
$$

and

$$
\sigma_{B \ell}^{p}=k \operatorname{Im}\left\{\frac{g_{\ell}^{\prime} G\left(z_{\ell}, z_{p}\right)}{U\left(z_{\ell}\right)-c} \frac{\hat{\eta}\left(z_{\ell}\right)}{\hat{\eta}\left(z_{p}\right)}\right\} .
$$

Note that for piecewise profiles a vorticity interface cannot cause growth in itself, i.e., $\sigma_{K p}^{p}=0$, since $\Delta q_{p}$ is a real number.

In deriving the partial growth rate formulation for smooth profiles we presume that the domain can be split into a number of interfacial regions, where either $U^{\prime \prime}$ or $N^{2}$ reaches some extrema. In the case of the $U^{\prime \prime}$ profile, the interfacial regions should also be separated by inflection points in the $U$ profile, i.e., zero crossings of $U^{\prime \prime}$. A $\hat{\psi}_{K, B}$ can then be defined for each interface, using Eq. (12), as

$$
\hat{\psi}_{K j}(z)=-\int_{D_{j}} G(s, z) U^{\prime \prime}(s) \hat{\eta}(s) d s,
$$

and

$$
\hat{\psi}_{B \ell}(z)=\int_{D_{\ell}} G(s, z) \frac{N^{2}(s)}{U(s)-c} \hat{\eta}(s) d s,
$$

where the $D_{j, \ell}$ denotes the domain of the $j$ th kinematic vorticity region and the $\ell$ th baroclinic vorticity region. Using Eq. (15), we can write

$$
\sigma=\sum_{j=1}^{n} \pi_{K j}(z)+\sum_{\ell=1}^{m} \pi_{B \ell}(z),
$$

with $\pi_{K j}(z)=k \operatorname{Im}\left(\hat{\psi}_{K j} / \hat{\eta}\right)$ and $\pi_{B \ell}(z)=k \operatorname{Im}\left(\hat{\psi}_{B \ell} / \hat{\eta}\right)$. Finally, to apply this condition to the $p$ th interfacial region, we multiply both sides of Eq. (B8) by a suitable weight function $F(z)$, integrate over $D_{p}$, and rearrange to give a direct analogy to Eq. (B3) for smooth profiles,

$$
\begin{aligned}
\sigma & =\sum_{j=1}^{n} \frac{\left\langle F \pi_{K j}\right\rangle_{p}}{\langle F\rangle_{p}}+\sum_{\ell=1}^{m} \frac{\left\langle F \pi_{B \ell}\right\rangle_{p}}{\langle F\rangle_{p}}, \\
& =\sum_{j=1}^{n} \sigma_{K j}^{p}+\sum_{\ell=1}^{m} \sigma_{B \ell}^{p},
\end{aligned}
$$

where \langle\rangle$_{p}$ indicates integration over $D_{p}$. A natural choice for the weight function is either $F=U^{\prime \prime}$ if $p$ corresponds to a vorticity interface, or $F=N^{2}$ if $p$ is a density interface, as has been used throughout the paper.

${ }^{1}$ H. Helmholtz, "On discontinuous movements of fluids," London, Edinburgh Dublin Philos. Mag. J. Sci. 36, 337 (1868).

${ }^{2}$ W. Kelvin, "Hydrokinetic solutions and observations," London, Edinburgh Dublin Philos. Mag. J. Sci. 42, 362 (1871).

${ }^{3}$ J. W. S. Rayleigh, "On the stability, or instability, of certain fluid motions," Proc. London Math. Soc. 12, 57 (1880).

${ }^{4}$ A. Michalke, "On the inviscid instability of hyperbolic-tangent velocity profile," J. Fluid Mech. 19, 543 (1964).

${ }^{5}$ J. Holmboe, "On the behavior of symmetric waves in stratified shear layers," Geofysiske Publikasjoner 24, 67 (1962).

${ }^{6} \mathrm{P}$. Hazel, "Numerical studies of the stability of inviscid stratified shear flows," J. Fluid Mech. 51, 39 (1972).

${ }^{7}$ L. N. Howard and S. A. Maslowe, "Stability of stratified shear flows," Boundary-Layer Meteorol. 4, 511 (1973).

${ }^{8}$ W. D. Smyth, G. P. Klaassen, and W. R. Peltier, "Finite amplitude Holmboe waves," Geophys. Astrophys. Fluid Dyn. 43, 181 (1988).

${ }^{9}$ W. D. Smyth and W. R. Peltier, "Instability and transition in finiteamplitude Kelvin-Helmholtz and Holmboe waves," J. Fluid Mech. 228, 387 (1991)

${ }^{10}$ A. McC. Hogg and G. N. Ivey, "The Kelvin-Helmholtz to Holmboe instability transition in stratified exchange flows," J. Fluid Mech. 477, 339 (2003).

${ }^{11} \mathrm{~S}$. A. Thorpe, "Experiments on instability and turbulence in a stratified shear flow," J. Fluid Mech. 61, 731 (1973).

${ }^{12}$ C. P. Caulfield and W. R. Peltier, "The anatomy of the mixing transition in homogenous and stratified free shear layers," J. Fluid Mech. 413, 1 (2000).

${ }^{13}$ W. D. Smyth, J. R. Carpenter, and G. A. Lawrence, "Mixing in symmetric Holmboe waves," J. Phys. Oceanogr. 37, 1566 (2007).

${ }^{14}$ J. R. Carpenter, G. A. Lawrence, and W. D. Smyth, "Evolution and mixing of asymmetric Holmboe instabilities," J. Fluid Mech. 582, 103 (2007).

${ }^{15}$ D. M. Farmer and L. Armi, "The flow of Mediterranean water through the strait of Gibraltar," Prog. Oceanogr. 21, 1 (1988).

${ }^{16}$ M. C. Wesson and M. C. Gregg, "Mixing at the Camarinal Sill in the strait of Gibraltar,” J. Geophys. Res. 99, 9847, doi:10.1029/94JC00256 (1994).

${ }^{17}$ S. Yoshida, M. Ohtani, S. Nishida, and P. F. Linden, "Mixing processes in a highly stratified river," Physical Processes in Lakes and Oceans, Coastal and Estuarine Studies Vol. 54 (American Geophysical Union, Washington, DC, 1998), pp. 389-400.

${ }^{18}$ E. W. Tedford, J. R. Carpenter, R. Pawlowicz, R. Pieters, and G. A. 
Lawrence, "Observation and analysis of shear instability in the Fraser River estuary," J. Geophys. Res. 114, C11006, doi:10.1029/ 2009JC005313 (2009).

${ }^{19}$ C. G. Koop and F. K. Browand, "Instability and turbulence in a stratified fluid with shear," J. Fluid Mech. 93, 135 (1979).

${ }^{20}$ G. A. Lawrence, F. K. Browand, and L. G. Redekopp, "The stability of a sheared density interface," Phys. Fluids 3, 2360 (1991).

${ }^{21}$ G. Pawlak and L. Armi, "Vortex dynamics in a spatially accelerating shear layer," J. Fluid Mech. 376, 1 (1998).

${ }^{22}$ C. P. Caulfield, W. R. Peltier, S. Yoshida, and M. Ohtani, "An experimental investigation of the instability of a shear flow with multilayered density stratification," Phys. Fluids 7, 3028 (1995).

${ }^{23}$ S. P. Haigh, "Non-symmetric Holmboe waves," Ph.D. thesis, University of British Columbia, 1995.

${ }^{24}$ G. A. Lawrence, S. P. Haigh, and Z. Zhu, "In search of Holmboe's instability," Physical Processes in Lakes and Oceans, Coastal and Estuarine Studies Vol. 54 (American Geophysical Union, Washington, DC, 1998), pp. 295-304.

${ }^{25}$ P. G. Baines and H. Mitsudera, "On the mechanism of shear flow instabilities," J. Fluid Mech. 276, 327 (1994).

${ }^{26}$ C. P. Caulfield, "Multiple linear instability of layered stratified shear flow," J. Fluid Mech. 258, 255 (1994).

${ }^{27}$ W. D. Smyth and K. B. Winters, "Turbulence and mixing in Holmboe waves," J. Phys. Oceanogr. 33, 694 (2003).

${ }^{28}$ R. A. Cairns, "The role of negative energy waves in some instabilities of parallel flows," J. Fluid Mech. 92, 1 (1979).

${ }^{29}$ L. G. Redekopp, "Elements of instability theory for environmental flows," Environmental Stratified Flows (Kluwer, Dordrecht, 2001).

${ }^{30}$ G. I. Taylor, "Effect of variation in density on the stability of superposed streams of fluid," Proc. R. Soc. London, Ser. A 132, 499 (1931).

${ }^{31}$ C. P. Caulfield et al. (Ref. 22) have found that it is possible for modes to form that are not at the wave number of maximum growth for a given set of parameters.

${ }^{32}$ A. Alexakis, "On Holmboe's instability for smooth shear and density profiles," Phys. Fluids 17, 084103 (2005).

${ }^{33}$ O. M. Umurhan and E. Heifetz, "Holmboe modes revisited," Phys. Fluids 19, 064102 (2007).

${ }^{34}$ S. Ortiz, J. M. Chomaz, and T. Loiseleux, "Spatial Holmboe instability," Phys. Fluids 14, 2585 (2002).

${ }^{35}$ K. B. Winters, J. A. MacKinnon, and B. Mills, "A spectral model for process studies of rotating, density-stratified flows," J. Atmos. Ocean. Technol. 21, 69 (2004). 